\title{
El papel de las variables relacionadas con las expectativas y la satisfacción en la eficacia del tratamiento de los trastornos adaptativos
}

\author{
IRYNA RACHYLA \\ rachyla@uji.es \\ OlAIA ROYO-GRAU \\ al189536@uji.es \\ MAR MOLÉS \\ molesm@uji.es \\ SOLEDAD QUERO \\ squero@uji.es
}

\section{Resumen}

Introducción: En la actualidad, los trastornos adaptativos (TA) constituyen uno de los problemas psicológicos más frecuentes en atención primaria y hospitalaria. No obstante, existen pocos estudios sobre su tratamiento y las variables que influyen en la eficacia del mismo. El objetivo de este trabajo es explorar el papel de las expectativas y la satisfacción en la eficacia del tratamiento de los TA. Método: La muestra incluyó 46 participantes con TA (34 mujeres y 12 hombres). La edad media fue de 33,28 años (d. $\mathrm{t} .=8,47$ ). Todos ellos recibieron un tratamiento cognitivo-conductual de 6-8 sesiones en 2 formatos (tradicional: $\mathrm{N}=22$ y realidad virtual: $\mathrm{N}=24$ ) y cumplimentaron las escalas de expectativas y satisfacción adaptadas de Borkovec y Nau (1972). Como medidas de eficacia se calcularon los índices de cambio fiable obtenidos tras el tratamiento y seguimiento a los 3 meses en el Inventario de Crecimiento Postraumático, el Inventario de Estrés y Pérdida (IEP) y la escala de afecto negativo del PANAS. Resultados: En el post-tratamiento, el cambio estuvo relacionado con la satisfacción de los pacientes con el tratamiento recibido, concretamente con los aspectos relacionados con su utilidad y lógica. En el seguimiento, además de la satisfacción, las expectativas resultaron ser también una variable importante cuando se utilizó el IEP como medida de cambio terapéutico. Conclusiones: Los resultados obtenidos ponen de manifiesto el papel que juegan las expectativas y satisfacción con el tratamiento en la eficacia terapéutica y, en consecuencia, la importancia de diseñar tratamientos psicológicos que sean no solamente eficaces, sino también bien aceptados por los pacientes.

Palabras clave: trastornos adaptativos, expectativas, satisfacción, eficacia terapéutica, índice de cambio fiable. 


\begin{abstract}
Introduction: Nowadays, $A D$ are one of the most prevalent psychological problems in primary care and hospital settings. However, few studies about its treatment and the variables that influence its efficacy are available. The aim of the present work is to explore the role of expectations and satisfaction in treatment efficacy of AD. Method: The sample included 46 participants with TA (34 women and 12 men). The mean age was 33.28 years $(S D=8.47)$. All they received $6-8$ cognitive-behavioral therapy sessions in 2 formats (traditional: $\mathrm{N}=22$ and virtual reality: $\mathrm{N}=24$ ) and filled out the expectations and satisfaction scales adapted Borkovec and Nau (1972). As measures of treatment outcome reliable change indexes were obtained after treatment and at 3-month follow-up for the Posttraumatic Growth Inventory, the Inventory of Stress and Loss (IES) and the PANAS negative affect scale. Results: At post-treatment, change was associated with participants' satisfaction with the received treatment, specifically with aspects related to its utility and logic. At follow-up, in addition to satisfaction, expectations turned out to be also an important variable when the IEL was used as therapeutic change measure. Conclusions: Results highlight the role of expectations and satisfaction with treatment in the therapeutic efficacy and, therefore, the importance of designing psychological treatments that are not only efficacious but also to be well accepted by patients.
\end{abstract}

Keywords: adjustment disorders, expectations, satisfaction, therapeutic efficacy, reliable change index.

\title{
Introducción
}

EI DSM-5 define los TA como una reacción emocional y comportamental negativa que interfiere en distintas áreas de la vida de la persona y aparece en respuesta a un estresor identificable dentro de los 3 meses siguientes a la aparición de éste (APA, 2013). Los clasifica en el capítulo "Trastornos relacionados con traumas y factores de estrés», junto con el trastorno de estrés postraumático y el trastorno de estrés agudo entre otros.

Alrededor de los TA se ha generado una situación bastante paradójica. Pues, si bien por un lado, constituyen un problema muy frecuente en la práctica clínica (Carta, Balestrieri, Murru y Hardoy, 2009), principalmente en contextos de atención primaria y hospitalaria, y están asociados con un deterioro social y laboral significativo, causando un alto porcentaje de bajas laborales (Van der Klink, Blonk, Schene y van Dijk, 2003); por otro, han recibido un escaso interés por parte de los investigadores. Como ponen de manifiesto Casey y Bailey (2011), se han llevado a cabo pocos estudios sobre el tratamiento de los TA, por lo que diversos autores se han centrado en establecer los principales objetivos a seguir en cualquier tratamiento para este problema y ofrecer directrices generales de tratamiento (e.g., Strain, 1995). En cosecuencia, los pocos estudios experimentales realizados han abordado el tratamiento de los TA desde muy diversas aproximaciones terapéuticas: terapia de espejo (González-Jaimes y TurnbullPlaza, 2003), terapia cognitivo-comportamental (TCC) (Van der Klink y cols., 2003), terapia psicodinámica (Itzhak y cols., 2012), entrenamiento en meditación (Srivastava, Talukdar y Lahan, 2011) o psicoterapia de cuerpo mente-espíritu (Hsiao y cols., 2014).

El grupo de Botella ha desarrollado un programa TCC específico para el tratamiento de los TA que incluye estrategias de psicología positiva (Botella, Baños y Guillén, 2008). Este 
protocolo puede aplicarse de forma tradicional o bien mediente la realidad virtual (RV). Concretamente para la aplicación del componente de elaboración del acontecimiento estresante se puede utilizar un dispositivo de RV flexible y adaptable denominado El Mundo de EMMA. Ya existen resultados preliminares de eficacia de este protocolo en dos estudios de caso (AndreuMateu, Botella, Quero, Guillén y Baños, 2012; Baños y cols., 2008), en una serie de 13 casos (Quero y cols., en prensa) y en un estudio entre grupos que incluyó participantes con diveros trastornos relacionados con el estrés (trastorno de estrés postraumatico, duelo complicado, y TA) en el que los programas de TCC aplicados de forma tradicional fueron igual de eficacies que los mismos programas pero apoyados por El Mundo de EMMA (Baños y cols., 2011).

Un siguiente paso es llevar a cabo estudios que exploren qué variables influyen en la eficacia del tratamiento. En este sentido, las expectativas de los pacientes han sido señaladas en la literaura desde hace más de 50 años como una de las variables que afectan al curso de la psicoterapia (Greenberg, Constantito y Bruce, 2006), aunque los resultados todavía no son igual de concluyentes para todos los trastornos mentales. Así, la investigación realizada con el trastorno obsesivo-compulsivo no encontró relación significativa entre las expectativas y la reducción de la sintomatología clínica en personas con este diagnóstico (Steketee y cols., 2011; Vogel, Hansen, Stiles y Gotestam, 2006). Sin embargo, dicha relación resultó ser positiva en un estudio realizado con pacientes con fobia a volar (Price, Anderson, Henrich y Rothbaum, 2008) y en otro con pacientes con fobia a volar, acrofobia y ansiedad social (Moldovan y David, 2014). En ambos trabajos se utilizó la RV como medio de exposición a los ambientes y estímulos temidos. En la misma línea, Price y Anderson (2012) aplicaron el mismo Tcc para miedo a hablar en público en dos formatos diferentes. A uno de los grupos se le aplicó el componente de exposición en grupo, mientras que los participantes del otro grupo realizaron la exposición mediante RV. En ambas condiciones las expectativas mostraron ser una variable predictora de cambio clínico, no habiendo diferencias significativas entre ellas. Resultados similares se obtuvieron en un trabajo realizado con pacientes con trastorno de pánico y agorafobia, en el que se compararon dos condiciones experimentales: exposición interoceptiva tradicional versus exposición interoceptiva mediante RV (Quero y cols., 2013).

No se ha encontrado en la literatura ningún estudio que explore la relación entre expectativas y la eficacia terapéutica para los TA. Por ello, el objetivo de este trabajo es explorar la relación entre tener unas altas expectativas respecto al tratamiento y una buena satisfacción con el mismo, con obtener mejores resultados mediante la aplicación de éste.

\section{Método}

\section{Participantes}

La muestra estaba compuesta por 46 participantes (34 mujeres y 12 hombres) diagnosticados de TA, según el DSM-IV-TR (APA, 2000). Todos ellos solicitaron ayuda en el Servicio de Asistencia Psicológica de la Universitat Jaume I de Castellón (España). La edad media fue de 33,28 años (d. t. $=8,47$ ), oscilando el rango de 18 a 50 . El 45,7 \% de la muestra estaba casada, el $39,1 \%$ casado o conviviendo con pareja y el $15,2 \%$ estaba separada/divorciada o viuda. En cuanto al nivel educativo, la mayoría $(76,1 \%)$ habían cursado estudios universitarios y el $23,9 \%$ tenían estudios primarios o secundarios.

Por lo que se refiere al subitpo de TA, la mayoría de los participantes $(78,3 \%)$ fueron diagnosticados de TA mixto con anisedad y depresión, seguidos por el TA con estado de ánimo depresivo (15,2\%), el TA con ansiedad (4,3\%) y el TA con alteración mixta de las emociones y el comportamiento $(2,2 \%)$. Los participantes incluidos en este estudio formaban parte de un 
estudio de eficacia controlado entre grupos que incluía 3 condiciones experimentales: TCC con $\mathrm{RV}(\mathrm{N}=15), \mathrm{TCC}$ tradicional $(\mathrm{N}=15)$ y grupo control lista de espera $(\mathrm{N}=16)$.

\section{Medidas}

A todos los participantes se les aplicó un protocolo de evaluación más amplio que incluía instrumentos específicamente diseñados por el equipo para evaluar los TA e instrumentos que recogen aspectos relacionados con el problema. En este trabajo solamente se incluyen las medidas relativas a las expectativas y satisfacción con el tratamiento y los cuestionarios utiizados para calcular el cambio clínicamente significativo (Jacobson y Truax, 1991).

Escala de expectativas y escala de satisfacción con el tratamiento (Adaptación de Borkovec y Nau, 1972). La Escala de expectativas se administró a los pacientes una vez se les había explicado la base lógica del tratamiento que iban a recibir, pero antes de que de que éste diera comienzo. La información que nos permite recoger esta escala es referente a la opinión/expectativa que el paciente se ha formado de dicho tratamiento. La Escala de satisfacción/opinión se aplicó una vez se había concluido el tratamiento y recogía las mismas cuestiones que las planteadas en la Escala de expectativas, pero esta vez el paciente las evaluaba teniendo en cuenta su experiencia con el tratamiento. Los aspectos que se recogen en ambas escalas se refieren a lógica del tratamiento; la satisfacción experimentada con el mismo; el grado en que se lo recomendaría a un amigo que tuviera el mismo problema; el grado en el que se considera que podría ser útil para tratar otros trastornos psicológicos; el grado en que se considera que será útil en el propio caso; y el grado en que consideran que la intervención será o ha sido aversiva.

Inventario de estrés y pérdida (IEP; adaptado de Prigerson y cols., 1995): se llevó a cabo una adaptación del ICG (Prigerson y cols., 1995) con el objetivo de utilizarlo en la evaluación de situaciones estresantes que estén interfiriendo de forma negativa en la vida de una persona. El instrumento es igual que el original con la excepción de que se sustituyeron las palabras que aluden a la persona fallecida por las que se refieren a la persona/situación que se ha perdido (e.g., pérdida de trabajo, pérdida de una pareja...). Evalúa la intensidad con la que interfiere la situación o persona perdida en la vida de la persona en una escala de 0 («nunca») a 4 («siempre»). A diferencia de la versión original del ICG, el IEP consta de 17 ítems (y no de 19 , ya que se eliminaron 2 ítems porque no eran adecuados para evaluar TA). Datos prelimnares de validación mostraron excelentes coeficientes de fiabilidad test-restest $(0,90)$ y de consistencia interna en población española tanto general $(0,91)$ como clínica con TA $(0,86)$ (Quero, Molés, Mor, Baños y Botella, 2014). Para los casos incluidos en este estudio cuyo acontecimiento estresante fue la muerte de un ser querido se utilizo el Inventario de Duelo Complicado de Prigerson y cols. (1995).

Inventario de crecimiento postraumático (PTGI; Tedeschi y Calhoun, 1996). Este instrumento evalúa los posibles cambios positivos que pueden experimentar las personas tras haber sufrido un acontecimiento traumático o adverso. Es una escala de 21 ítems, puntuados de 0 («No experimenté este cambio como resultado de la crisis») a 6 («Experimenté este cambio en medida muy grande como resultado de la crisis»). Puntuaciones elevadas en este inventario indican un mayor grado de crecimiento postraumático percibido.

Escala de afecto positivo y negativo (Watson, Clark y Tellegen, 1988). Se trata de un cuestionario de 20 ítems que evalúa las dimensiones positiva (e.g., interesado, ilusionado, satisfecho) y negativa del afecto (e.g., angustiado, culpable, agresivo). En este trabajo solo se analizó la escala de afecto negativo. Los participantes tenían que valorar cada ítem en una escala de 1 a 5 (1=nada o muy ligeramente; $5=$ mucho) indicando el grado en el que cada ad- 
jetivo describía mejor el estado de ánimo en el que se encontraban en el momento de responder al cuestionario.

\section{Tratamiento}

Todos los participantes recibieron un tratamiento cognitivo-conductual (Botella, Baños y Guillén, 2008) que contaba con un número variable de sesiones (entre 6 y 8), dependiendo de la situación clínica de cada paciente. Las sesiones eran de 90 minutos de duración y se realizaban con periodicidad semanal. Los componentes terapéuticos incluidos fueron: psicoeducación (una sesión), aceptación y elaboración del acontecimiento negativo (4-6 sesiones) y prevención de recaídas (una sesión). También se incluyeron estrategias de psicología positiva con la finalidad de aumentar la capacidad natural del ser humano para resistir y crecer ante la adversidad (Peterson y Seligman, 2004) y se utilizaron, por una parte, ejercicios dirigidos a extraer aspectos positivos a partir de lo vivido, basados en Neimeyer (2000) y, por otra, instruir en el afrontamiento de los problemas. Ahora bien, no se sigue el enfoque de D'Zurilla y Goldfried (1971) basado en dimensiones pues lo que se pretende es que el paciente llegue a considerar los problemas como algo absolutamente necesario para crecer y progresar.

Todos los participantes recibirieron el mismo protocolo de TCC, pero aproximadamente la mitad de ellos recibió el componente de elaboración mediante $\mathrm{RV}(\mathrm{N}=24)$ y la otra mitad de forma tradicional cara a cara $(\mathrm{N}=22)$. Como se ha mencionado anteriormente, se utilizó el sistema de RV El Mundo de EMMA, un ambiente virtual flexible que permite realizar una representación física de los significados y emociones que la persona asocia a diferentes situaciones problemáticas. El usuario puede controlar el aspecto de El Mundo de EMMA eligiendo entre 5 escenarios diferentes (un desierto, una playa, un bosque de zarzas, un paisaje nevado o un prado). Estos escenarios pueden ser personalizados manipulando diferentes efectos (e.g., el momento del día, la lluvia, la nieve, la tormenta con rayos y truenos, etc.) y su intensidad. Además, El Mundo de EMMA dispone de un conjunto de símbolos, imágenes, música y colores, a través de los cuales el usuario puede describir y representar el acontecimiento adverso. Gracias a estas propiedades, es posible generar ambientes clínicamente significativos para cada participante, capaces de evocar acontecimientos traumáticos diferentes (e.g., ruptura sentimental, enfermedad, problemas laborales, etc.). Una descripción más detallada de El Mundo de EMMA se puede encontrar en Baños y cols. (2011) y Quero y cols. (en prensa).

\section{Procedimiento}

Todos los participantes realizaron una entrevista de screening, seguida de dos sesiones de evaluación. Los participantes que cumplieron con los criterios de inclusión firmaron el consentimiento informado para participar en el estudio. En la primera sesión de evaluación se confirmó el diagnostico de TA y se completaron las diferentes medidas de autoinforme. En la segunda sesión de evaluación se establecieron los objetivos terapéuticos. Finalizada la fase de evaluación, las personas consideradas aptas para el estudio fueron asignadas al azar a una de las tres condiciones experimentales (TCC RV, TCC Tradicional y grupo control lista de espera) utilizando, el programa informático «RandomAlloc». A continuación, las personas asignadas a las dos condiciones de tratamiento recibieron de 6 a 8 sesiones de intervención. Por su parte, tras finalizado el periodo de lista de espera, los participantes incluidos en este grupo fueron asignados a una de las dos condiciones de tratamiento: 7 fueron asignados a la condición tradicional y 9 a la condición de RV. Todos los participantes fueron evaluados de nuevo al 
finalizar el tratamiento y a los 3 meses de seguimiento. En la aplicación de la evaluación y el tratamiento participaron un total de 5 terapeutas pertenecientes al Servicio de Asistencia Psicológica de la Universitat Jaume I. Todos ellos contaban con experiencia en la aplicación de programas de tratamiento de orientación cognitivo-conductual y en técnicas de RV y estuvieron supervisados por psicólogos clínicos expertos.

\section{Resultados}

Con el fin de analizar la capacidad de las variables relacionadas con expectativas y satisfacción para predecir la eficacia del tratamiento de los TA, se realizó un análisis de regresión lineal por pasos. Las escalas de expectativas y satisfacción fueron utilizadas como variables independientes, mientras que como variables dependientes se tomaron los índices de cambio fiable (ICF) de Jacobson y Truax (1991) calculados para las medidas PTGI, IEP y PANAS- en el post-tratamiento y el seguimiento a los 3 meses. El cambio clínicamente significativo obtenido a partir de los ICF para cada medida de eficacia terapéutica se puede consultar en la tabla 1.

Tabla 1

Cambio clínicamente significativo para PTGI, IEP y PANAS- en post-tratamiento y el seguimiento de los 3 meses

\begin{tabular}{lcccccc}
\hline & \multicolumn{2}{c}{ PTGI } & \multicolumn{2}{c}{ IEP } & \multicolumn{2}{c}{ PANAS- } \\
\hline & Post & $\begin{array}{c}\text { Seguimien- } \\
\text { to 3 meses }\end{array}$ & Post & $\begin{array}{l}\text { Seguimien- } \\
\text { to 3 meses }\end{array}$ & Post & $\begin{array}{c}\text { Seguimien- } \\
\text { to 3 meses }\end{array}$ \\
\hline$n(\%)$ & $n(\%)$ & $n(\%)$ & $n(\%)$ & $n(\%)$ & $n(\%)$ \\
\hline Recuperado & $4(8,9 \%)$ & $6(15 \%)$ & $24(54,5 \%)$ & $25(69,4 \%)$ & $20(44,4 \%)$ & $20(51,3 \%)$ \\
\hline Mejorado & $15(33,3 \%)$ & $10(25 \%)$ & $0(0 \%)$ & $0(0 \%)$ & $0(0 \%)$ & $1(2,6 \%)$ \\
\hline No cambio & $26(57,8 \%)$ & $24(60 \%)$ & $20(45,5 \%)$ & $11(30,6 \%)$ & $25(55,6 \%)$ & $18(46,2 \%)$ \\
\hline
\end{tabular}

Como se puede observar en la tabla 2, en el post-tratamiento el cambio estuvo relacionado únicamente con la satisfacción de los pacientes con el tratamiento recibido, concretamente con aspectos relacionados con el grado en el que los participantes percibían el tratamiento recibido como útil para su problema, lógico y aversivo.

En el seguimiento a los 3 meses, la satisfacción con el tratamiento continuaba siendo una variable importante. De nuevo se encontró que el grado en el que los participantes percibían el tratamiento como lógico y útil para su problema influía en el cambio que presentaban al finalizar el programa de intervención. A estas dos variables se añadió el ítem que medía el grado en el que los participantes recomendarían el tratamiento recibido a otras personas. Además, en este momento de evaluación, cuando se consideró el IEP como medida de cambio terapéutico, se observó que las expectativas también predecían la mejoría clínica. Concretamente, el cambio se relacionó con el grado en el que los participantes se sintieron satisfechos con el tratamiento que se les había propuesto para solucionar su problema y el grado en el que recomendarían dicho tratamiento a otras personas con un problema parecido al suyo.

Por último, las variables de satisfacción y expectativas alcanzaron mayor capacidad de predicción cuando se utilizó IEP como instrumento de medida de cambio clínicamente significativo. 
Tabla 2

Análisis de regresión por pasos entre las escalas de expectativas y satisfacción y el índice de cambio fiable para PTGI, IEP y PANAS- en el post-tratamiento y seguimiento a los 3 meses

\begin{tabular}{|c|c|c|c|c|c|}
\hline \multicolumn{2}{|c|}{ Post-tratamiento } & \multirow{2}{*}{$\begin{array}{c}\mathrm{R} 2 \\
0,32\end{array}$} & \multirow{2}{*}{$\begin{array}{c}\beta \\
0,58\end{array}$} & \multirow{2}{*}{$\begin{array}{c}t \\
4,07\end{array}$} & \multirow{2}{*}{$\frac{p}{0,000^{* *}}$} \\
\hline PTGI & $\begin{array}{l}\text { Satisfacción: ítem utilidad del tratamiento } \\
\text { para su problema }\end{array}$ & & & & \\
\hline \multirow{3}{*}{ IEP } & Satisfacción: ítem lógica del tratamiento & & $-0,55$ & $-3,74$ & $0,001^{* *}$ \\
\hline & & 0,34 & & & \\
\hline & Satisfacción: ítem aversividad del tratamiento & & $-0,40$ & $-2,76$ & $0,010^{*}$ \\
\hline PANAS- & $\begin{array}{l}\text { Satisfacción: ítem utilidad del tratamiento } \\
\text { para su problema }\end{array}$ & 0,13 & $-0,40$ & $-2,44$ & $0,021^{*}$ \\
\hline \multicolumn{6}{|c|}{ Seguimiento 3 meses } \\
\hline \multirow[t]{2}{*}{ PTGI } & $\begin{array}{l}\text { Satisfacción: ítem utilidad del tratamiento } \\
\text { para su problema }\end{array}$ & 0,32 & 0,58 & 3,97 & $0,000^{* *}$ \\
\hline & Satisfacción: ítem lógica del tratamiento & & $-0,31$ & $-2,03$ & 0,052 \\
\hline \multirow[t]{2}{*}{ IEP } & Expectativas: ítem recomendaría el tratamiento & 0,43 & 0,68 & 3,62 & $0,001^{* *}$ \\
\hline & Expectativas: ítem satisfacción & & $-0,57$ & $-2,82$ & $0,009^{* *}$ \\
\hline PANAS- & Satisfacción: ítem recomendaría el tratamiento & 0,20 & $-0,47$ & $-2,93$ & $0,006^{* *}$ \\
\hline
\end{tabular}

${ }^{* *} \rho<0,01 ;{ }^{*} \rho<0,05$

\section{Discusión y conclusiones}

El objetivo de este trabajo fue explorar el papel de las expectativas y la satisfacción en la eficacia del tratamiento de los TA. Los resultados obtenidos revelaron que, al finalizar el tratamiento, la satisfacción con el mismo predecía la mejoría clínica. Se observó que el hecho de valorar el tratamiento recibido como útil y lógico para tratar el problema para el que se había utilizado y, además, percibirlo como poco aversivo, predecía la reducción de la sintomatología clínica. Por otro lado, en el seguimiento a los 3 meses, la satisfacción con el tratamiento continuaba prediciendo el cambio terapéutico. Aquellos participantes que valoraron el tratamiento como útil y lógico para su problema e indicaron que se lo recomendarían a otras personas mostraron un mayor cambio respecto al inicio de tratamiento. Además, en este momento de evaluación las expectativas también predecían el cambio terapéutico, cocretamente en cuanto a la satisfacción percibida con respecto al tratamiento que iban a recibir y el grado en el que lo recomendarían a otras personas con su mismo problema.

Estos resultados van en la misma línea de otras investigaciones que sugieren la importancia de las expectativas en la eficacia de las psicoterapias (Glass, Arnkoff y Shapiro, 2001; Greenberg y cols., 2006). También son consistentes con los resultados de otros trabajos que han utilizado tratamientos apoyados en sistemas de RV (Moldovan y David, 2014; Price y cols., 2008). Sin embargo, no se ha encontrado en la literatura ningún estudio que haya investigado el papel de las expectativas en el tratamiento de los TA. Además, tampoco se ha encontrado ningún estudio que explore la importancia de la satisfacción con el tratamiento en la eficacia del mismo para este trastorno mental. El único estudio similar encontrado ha sido realizado con población con trastorno de pánico y agorafobia (Quero y cols., 2013). En este trabajo se 
encontró que la satisfacción junto con las expectativas predecían la mejoría terapeutica en el post-tratamiento, mientras que en el seguimiento a los 3 meses solamente las expectativas coninuaban prediciendo dicha mejoría. Por consiguiente, el presente trabajo aporta información novedosa en cuanto a factores que influyen en los resultados de los tratamientos psicológicos, específicamente de los TA, un diagnóstico clínico prevalente pero escasamente investigado por la comunidad científica.

Otro hallazgo a subrayar es el referente a los instrumentos de medida de eficacia terapéutica. De los instrumentos utlizados, el IEP fue la medida en la que más cambio se observó en los participantes. Además, se encontró que la satisfacción y, sobre todo, las expectativas predecían mejor la eficacia del tratamiento cuando se tomaba el IEP como instrumento de medida del cambio clínico, tanto en el post-tratamiento como en el seguimiento a los 3 meses. Esto no es de extrañar dado que se trata de un instrumento de evaluación especifico para los TA.

Para finalizar, es importante señalar que los resultados de este trabajo deben tomarse con cautela, pues presenta limitaciones. La más importante es la que se refiere al pequeño tamaño de la muestra incluida, teniendo en cuenta el gran número de variables independientes utilizadas en el análisis de regresión. No obstante, a pesar de esta limitación, los resultados obtenidos son sin duda innovadores y aportan conocimiento acerca de las variables que explican la eficacia en este caso del TCC (tradicional o apoyado con RV) para los TA.

En conclusión, los resultados obtenidos ponen de manifiesto el papel que juegan las expectativas y satisfacción con el tratamiento en la eficacia terapéutica y, en consecuencia, la importancia de diseñar tratamientos psicológicos no solamente eficaces, sino también que sean bien aceptados por los pacientes. Hacen falta más estudios que sigan aportando evidencias en este campo para, de esta forma, poder proporcionar un servicio más adecuado a las personas que lo necesitan, alcanzar mejores resultados terapéuticos y reducir el porcentaje de abandonos en los tratatamientos psicológicos.

\section{Referencias bibliográficas}

American Psychiatric Association (APA). (2000). Diagnostic and statistical manual of mental disorders DSM-IV-TR ( $4^{\text {th }}$ ed., text revision). Washington, DC: APA.

American Psychiatric Association (APA). (2013). Diagnostic and statistical manual of mental disorders DSM-V (5 th ed.). Washington, DC: APA.

Andreu-Mateu, S., Botella, C., Quero, S., Guillén, V. y Baños, R. (2012). La utilización de la realidad virtual y estrategias de psicología positiva en el tratamiento de los trastornos adaptativos. Behavioral Psychology/Psicología Conductual, 20, 323-348.

Baños, R. M., Botella, C., Guillén, V., García-Palacios, A., Jorquera, M. y Quero, S. (2008). Un porgrama de tratamiento para los trastornos adaptativos. Un estudio de caso. Apuntes de Psicología, 26, 303-316.

Baños, R. M., Guillén, V., Quero, S., García-Paracios, A., Alcaniz, M. y Botella, C. (2011). A virtual reality system for the treatment of stress-related disorders: A preliminary analysis of efficacy compared to a standard cognitive behavioral program. International Journal of Human-Computer Studies, 69, 602-613.

Ben-Itzhak, S., Bluvstein, I., Schreiber, S., Aharonov-Zaig, I., Maor, M., Lipnik, R., y Bloch, M. (2012). The Effectiveness of Brief Versus Intermediate Duration Psychodynamic Psychotherapy in the Treatment of Adjustment Disorder. Journal of Contemporary Psychotherapy, 42, 249-256.

Borkovec, T. D. y Nau, S. D. (1972). Credibility of analogue therapy rationales. Journal of Behavior Therapy and Experimental Psychiatry, 3, 257-260. 
Botella, C., Baños, R. M. y Guillén, V. (2008). Una propuesta de tratamiento para los trastornos adaptativos: creciendo en la adversidad. En C. Vázquez y G. Hervás (eds.), Psicología Positiva aplicada (pp. 129-154). Bolbao: Desclée de Brouwer.

Carta, M. G., Balestrieri, M., Murru. A. y Hardoy, M. C. (2009). Adjustment Disorder: epidemiology, diagnosis and treatment. Clinical Practice and Epidemiology in Mental Health, 5, $1-15$.

Casey, P. y Bailey, S. (2011). Adjustment disorders: the state of the art. World Psychiatry, 10, 11-18.

D'Zurilla, T. S. y Goldfried, M. R. (1971). Problem solving and behavior modification. Journal of Abnormal Psychology, 73, 117-126.

Glass, C. R., Arnkoff, D. B. y Shapiro, S. J. (2001). Expectations and preferences. Psychotherapy: Teory, Research, Practice, Training, 38, 455-461.

González-Jaimes, E. I. y Turnbull-Plaza, B. (2003). Selection of Psychotherapeutic Treatment for Adjustment Disorder with Depressive Mood Due to Acute Myocardial Infarction. Archives of Medical Research, 34, 924-932.

Greenberg, R. P., Constantino, M. J. y Bruce, N. (2006). Are patient expectations still relevant for psychotherapy process and outcome? Clinical Psychology Review, 26, 657-678.

Hsiao, F. H., Lai, Y. M., Chen, Y. T., Yang, T. T., Liao, S. C., Ho, R. T. H., Ng, S. M., Jow, G. M. (2014). Efficacy of psychotherapy on diurnal cortisol patterns and suicidal ideation in adjustment disorder with depressed mood. General Hospital Psychiatry, 36, 214-219.

Jacobson, N. S. y Truax, P. (1991). Clinical significance: A statistical approach to defining meaningful change in psychotherapy research. Journal of Consulting and Clinical Psychology, 59, 12-19.

Moldovan, R. y David, D. (2014). One session treatment of cognitive and behavioral therapy and virtual reality for social and specific phobias: Preliminary results from a randomized clinical trial. Journal of Cognitive and Behavioral Psychotherapies, 14, 67-83.

Neimeyer, R. A. (2000). Lessons of Loss. Florida: Keystone Heights.

Peterson, C. y Seligman, M. E. (2004). Character strengths and virtues: A handbook and classification. American Psychological Association, Washington, DC.

Price, M. y Anderson, P. L. (2012). Outcome expectancy as a predictor of treatment response in cognitive behavioral therapy for public speaking fears within social anxiety disorder. Psychotherapy, 49, 173-179.

Price, M., Anderson, P., Henrich, C. C. y Rothbaum, B. O. (2008). Greater expectations: Using hierarchical linear modeling to examine expectancy for treatment outcome as a predictor of treatment response. Behavior Therapy, 39, 398-405.

Prigerson, H. G., Maciejewsku, P. K., Reynolds, C. F., Bierhals, A. J., Newsom, J. T., Fasiczka, A. y cols. (1995). Inventory of Complicated Grief: A scale to measure maladaptive symptoms of loss. Psychiatry Research, 59, 65-79.

Quero, S., Andreu-Mateu, S., Moragrega, I., Baños, R. M., Molés, M., Nebot, S. y Botella, C. (en prensa). Un programa cognitivo-conductual que utiliza la realidad virtual para el tratamiento de los trastornos adaptativos: Una serie de casos. Revista Argentina de Psicología Clínica.

Quero, S., Molés, M., Mor, S., Baños, R. M. y Botella, C. (2014). Spanish validation of the complicated grief inventory adapted for adjustment disorders. ABCT $48^{\text {th }}$ Annual Convention.

Quero, S., Pérez-Ara, M. A., Bretón-López, J. M., García-Palacios, A., Baños, R. M. y Botella, C. (2013). Acceptability of virtual reality interoceptive exposure for the treatment of panic disorder with agoraphobia. British Journal of Guidance \& Counselling, 42, 123-137. 
Srivastava, M., Talukdar, U. y Lahan, V. (2011). Meditation for the management of adjustment disorder anxiety and depression. Complementary Therapies in Clinical Practice, 17, 241245.

Steketee, G., Siev, J., Fama, J. M., Keshaviah, A., Chosak, A. y Wilhelm, S. (2011). Predictors of treatment outcome in modular cognitive therapy for obsessive-comuplsive disorder. Depression and Anxiety, 28, 333-341.

Strain, J. J. (1995). Adjustment disorders. En G. O. Gabbard (ed.), Treatments of Psychiatric

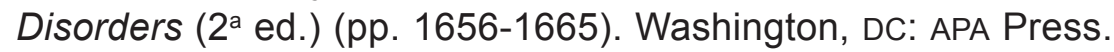

Tedeschi, R. G. y Calhoun, L. G. (1996). The Posttraumatic Growth Inventory: Measuring the Positive Legacy of Trauma. Journal of Traumatic Stress, 9, 455-471.

Van der Klink, J. L., Blonk, R. W., Schene, A. H. y van Dijk, F. J. (2003). Reducing long term sickness absence by an activating intervention in adjustment disorders. Occupational and Environmental Medicine, 60, 429-437.

Vogel, P. A., Hansen, B., Stiles, T. C. y Gotestam, K. G. (2006). Treatment motivation, treatment expectancy, and helping alliance as predictors of outcome in cognitive behavioral treatment of OCD. Journal of Behavior Therapy and Experimental Psychiatry, 37, 247255.

Watson, D., Clark, L. A. y Tellengen, A. (1988). Development and validation of brief measures of positive and negative affect: the PANAS scales. Journal of Personality and Social Psychology, 54, 1063-1070. 